\title{
La "viva speranza" di Dante e il problema della salvezza dei pagani virtuosi. Una lettura di Paradiso 20**
}

1. I canti del cielo di Giove (Paradiso 17-20) sono dedicati alla trattazione della grande tematica della Salus. Questa tematica soteriologica Dante però non la intende più nella sola prospettiva terrena e mondana, secondo il modello della poesia delle "armi" di Bertran de Born, come faceva nel De vulgari eloquentia; ma la trascrive ricopiandola direttamente dal Libro che contiene la parola divina, secondo cioè il modello analogico scritturale, in una prospettiva diventata ormai eterna. Dall'alta specola della sfera paradisiaca Dante può cosí offrire al suo lettore una definitiva soluzione, teorica e pratica, del problema della giustizia: sia del funzionamento della giustizia divina (problematica che sta al centro dell'inventio del poema sacro), sia della realizzazione e dei limiti della giustizia umana. Significativamente Beatrice, condensazione delle tematiche della Venus e della Virtus, rimane assente dall'azione narrativa, e non partecipa alla discussione ideologica che prende sviluppo in questi canti. Il suo posto viene preso dalla "bella image" dell'aquila: una sorta di arci-personaggio col quale l'actor si confronta, e nel quale compiutamente si ritrova. ${ }^{1}$

Il canto 20, che qui direttamente ci interessa, ripropone in modo chiasmatico la strutturazione del canto che lo precede. In tutt'e due i canti la materia si organizza infatti attorno a due discorsi pronunciati dall'aquila: ma mentre nel canto $19 \mathrm{il} \mathrm{primo} \mathrm{discorso} \mathrm{è} \mathrm{centrato} \mathrm{sulla}$ questione teorica e teologica della giustizia divina, e il secondo sulla questione pratica e storica dell'ingiustizia umana (si offrono exempla della presente corruzione della giustizia nel mondo cristiano); nel canto 20 invece il primo discorso considera degli exempla positivi di giustizia umana (tutti però proiettati verso un passato archetipico e mitico), e il secondo affronta il problema teorico della giustizia 
divina, del quale propone due codicilli (i due casi-limite relativi alla sorte eterna di Traiano e Rifeo).

I nodi tematico-ideologici del 20 canto del Paradiso si trovano incuneati dentro le due partizioni fondamentali del testo che abbiamo ora evidenziato. Il primo nodo è costituito dal problema della continuità/discontinuità fra mondo pagano e mondo cristiano, e rappresenta la cristallizzazione di una tematica più generale, quella del ritrovamento del filo conduttore della storia umana (tematica svolta soprattutto nel primo discorso dell'aquila: vv. 31-72); il secondo nodo è invece formato dal problema della salvazione dei pagani virtuosi, e rappresenta la punta di diamante della grave questione teologica della predestinazione divina (questione agitata nel secondo discorso dell'aquila: vv. 88-132). Cercheremo di sciogliere questi nodi all'interno di una lectura articolata del testo dantesco. ${ }^{2}$

I sei principi giusti che formano la parte più nobile dell'aquila, l'occhio, sono presentati, nel primo discorso del "santo uccello," nel rispetto assoluto delle proporzioni testuali (due terzine per ogni personaggio) e secondo una rigorosa struttura anaforica, per mezzo della quale viene enfatizzata l'opposizione fra passato terreno e verità parziale (prima terzina), e presente celeste e verità totale (seconda terzina). Essi si distribuiscono anche in misura uguale fra principi che vissero prima di Cristo (David, Rifeo, Ezechia) e principi che vissero dopo Cristo (Traiano, Costantino, Guglielmo II); ciò che crea una linea di separazione ideale fra i due gruppi. A segnare il confine sta l'evento capitale nella storia dell'uomo: l'Incarnazione di Cristo. È a partire da questo momento, infatti, che il tessuto dei rapporti fra l'uomo e Dio, lacerato dalla Colpa, ha potuto essere ricucito; e che l'immagine divina dell'uomo, oscurata dal peccato originale, ha potuto essere restaurata. Di qui la giustificazione ideologica del rinvenimento, in due punti identici dei canti 19 e 20 (ai vv. 103-5), dell'allusione alla fede nella Passione di Cristo, inizio necessario della reintegrazione umana nella condizione divina: "A questo regno / non salí mai chi non credette 'n Cristo, / né pria né poi ch'el si chiavasse al legno"; "D'i corpi suoi non uscir, come credi, / Gentili, ma Cristiani, in ferma fede / quel d'i passuri e quel d'i passi piedi."3

Il canone dei principi giusti intende offrirci, in una prospettiva fortemente raccorciata, la storia dell'imperium christianum, nei suoi 
archetipi, biblico (David e Ezechia) e classico (Rifeo), e nelle sue tappe evolutive essenziali, romana (Traiano), romano-cristiana (Costantino) e feudale-cavalleresca (Guglielmo II). Preliminari alla nostra indagine del canto sono l'accertamento delle motivazioni sottostanti alla selezione di tali nomi, e la spiegazione del loro inserimento in questo punto particolare del viaggio salvifico.

Se ideologicamente scontata è la scelta di David ad occupare il posto d'onore (la "pupilla" dell'occhio dell'aquila) in questa élite principesca (egli rappresenta infatti la radix Jesse dalla quale dovrà uscire la virga di Maria e il flos di Cristo), meno scontata è la funzione testuale del suo inserimento:

Colui che luce in mezzo per pupilla, fu il cantor de lo Spirito Santo, che l'arca traslatò di villa in villa:

ora conosce il merto del suo canto, in quanto effetto fu del suo consiglio, per lo remunerar ch'è altrettanto.

Le opere terrene che stanno alla base del "merto" di David ("merto" sul quale si è posata l'illuminazione divina) sono politico-religiose ("l'arca traslatò di villa in villa"), ${ }^{5}$ e soprattutto poetiche ("fu il cantor de lo Spirito Santo"): e andrà enfatizzato il fatto che Dante fa qui riferimento alla scrittura letteraria ("in quanto effetto fu del suo consiglio"), non alla scrittura ispirata del libro sacro, all'anctor umano non all'Auctor divino. ${ }^{6}$ È proprio a proposito di questo "canto" umano, canto di penitenza per i propri peccati e di lode a Dio per la sua misericordia; è proprio a proposito di questa storia di un'anima che dall'abisso del peccato si innalza alla vetta della grazia, che si crea un evidente parallelismo con il "canto" della Commedia, con la storia del viator che dalla selva infernale si è innalzato fino alla visione di Dio.

Ad un'esigenza profondamente autogiustificativa sembra rispondere anche l'inserzione del terzo spirito, Ezechia (sul secondo, Traiano, cosí come sull'ultimo, Rifeo, dovremo sostare più a lungo nella parte finale della nostra lectura):

E quel che segue in la circunferenza di che ragiono, per l'arco superno, morte indugiò per vera penitenza: 
ora conosce che 'l giudicio etterno non si trasmuta, quando degno preco fa crastino là giú de l'odierno.

Immotivati appaiono i dubbi dell'esegesi moderna sulla validità dell'identificazione del terzo "fuoco" dell'occhio dell'aquila con il giusto e saggio re d'Israele, Ezechia: identificazione basata soprattutto su un capitolo di Isaia, il trentottesimo, che è vitale anche per la fictio globale del poema sacro. La vita di Ezechia si divide nel racconto biblico in due parti: quella che ha già trascorso in una condizione di malattia, e quella che gli resta ancora da vivere, se gli verrà concessa la guarigione; al centro sta il paventato descensus ad inferos, il viaggio nel regno della morte: "Ego dixi: in dimidio dierum meorum vadam ad portam inferi" (38.10); viaggio rinviato per quindici anni a causa dell'intervento divino propiziato dal profeta Isaia. Anche la vita del viator appare divisa nella Commedia in due parti: la prima metà vissuta nella regio dissimilitudinis, la seconda metà nella regio similitudinis; al centro si pone il temuto descensus ad inferos, il viaggio nel regno della morte spirituale: "Nel mezzo del cammin di nostra vita / mi ritrovai per una selva oscura"; viaggio che il protagonista vorrebbe rinviare, ma la cui necessità è affermata da Virgilio ("a te convien tenere altro viaggio"), e la cui realizzazione è predisposta da Dio: attraverso tale catabasi infatti potrà essere scongiurata non la morte terrena, bensì quella eterna del poeta-pellegrino e dell'umanità che egli rappresenta.

La funzionalità del successivo medaglione dedicato a Costantino (lo storico conciliatore dei diritti dell'Impero con i privilegi della Chiesa) sembra risedere non nel rispecchiamento della biografia letteraria del poeta (come David, Ezechia, e poi Rifeo), ma nell'occasione che esso offre all'actor di pronunciare una palinodia rispetto a giudizi precedentemente formulati sul conto dell'imperatore romano:

L'altro che segue, con le leggi e meco, sotto buona intenzion che fé mal frutto, per cedere al pastor si fece greco:

ora conosce come il mal dedutto dal suo bene operar non li è nocivo, avvegna che sia 'l mondo indi distrutto. 
Naturalmente è qui questione della famosá Donatio Constantini (dell'alienazione cioè di poteri di esclusiva giurisdizione imperiale in favore della Chiesa), sulla quale la riflessione dantesca si era già in precedenza dedicata. Nel De Monarchia in particolare Dante aveva manifestato (accetto la datazione nardiana del trattato politico) ${ }^{7}$ un moto di aperta sfiducia nei confronti della provvidenzialità e della giustizia divine, allorquando si era rivolto ad analizzare questo episodio-chiave dei rapporti fra Chiesa e Impero: "o felicem populum, o Ausoniam te felicem, si vel numquam infirmator ille Imperii tui natus fuisset, vel numquam sua pia intentio [cfr. v. 56] ipsum fefellisset" (2.11.8); la ratio umana, se avesse potuto, avrebbe voluto strappare quella pagina funesta dalla storia del mondo cristiano. Lo spirito col quale lo stesso evento viene trattato nel Paradiso è totalmente cambiato: vista dalla specola della fides, illuminata dalla luce della grazia, la donazione, benché apportatrice di tristissime conseguenze storiche ("avvegna che sia 'I mondo indi distrutto"), appare perfettamente accettabile, in quanto facente parte della superiore programmazione divina, imperscrutabile dagli uomini e non totalmente accessibile neanche agli stessi beati; ciò che conta ora, nella prospettiva eterna, è la "buona intenzion," il "ben operar," non il "mal dedutto" da azioni compiute obbedendo al volere divino.

Del lungo periodo che intercorre fra la stabilizzazione dei rapporti dell'Impero con la Chiesa e l'epoca moderna, una sola figura di principe giusto viene qui celebrata: quella del re normanno Guglielmo II, il quale favorendo le nozze di Costanza d'Altavilla con Enrico VI di Svevia aveva propiziato la nascita dell'"ultima possanza" della casa sueva, dell'imperatore Federigo II (cfr. Par. 3.118-20):

E quel che vedi ne l'arco declivo, Guiglielmo fu, cui quella terra plora che piagne Carlo e Federigo vivo:

ora conosce come s'innamora lo ciel del giusto rege, e al sembiante del suo fulgore il fa vedere ancora.

Guglielmo II, vissuto nella seconda metà del secolo XII, all'epoca cioè della massima fioritura della civiltà cortese, è delegato a dar vita all'ultima vibrazione nel poema sacro di quel mondo definitivamente tramontato, ma anche insistentemente rimpianto, delle "donne 
antiche e' cavalieri." La sua fama di principe cortese doveva essere corrente, se la Cronica fiorentina falsamente attribuita a Brunetto Latini può affermare di lui: ". . . Guiglielmo . . . in tucti suoi facti fu savio e gratioso sopra gli altri principi del mondo a quel tempo. Nel costui tempo il regnio di Puglia e di Cicilia crebbe e abondò di richeççe e d'allegramento e di gaudio e letitie, più che nullo altro reame del mondo: ché questo re Guiglielmo li teneva in tanta pace, ch'elli non actendeano se none a sonare e ad cantare e dançare. Et quasi elli fecero di nuovo un'altra Tavola Ritonda." ${ }^{8}$ Brani cronachistici o narrazioni novellistiche di questo tipo sono quelli che hanno certo fornito a Dante la base documentaria per proclamare Guglielmo specchio perfettissimo del mondo cavalleresco. Basta, in realtà, riflettere sull'opposizione plora/piagne per rendersi conto che questa è una spia linguistica della dicotomia valori cortesi/valori borghesi: se infatti il raffinato latinismo plora dà voce all'accorato rimpianto per quell'epoca mitica definitivamente scomparsa, il termine volgare piagne ha il valore di configurare l'aspra rampogna contro i contemporanei reggitori di quella parte dell'Italia, "Carlo [II d'Angiò] e Federigo [II d'Aragona]." D'altronde l'impiego del verbo "s'innamora," a descrivere l'amore divino per l'anima eletta, sembra definitivamente consegnare questo termine-cardine della tematologia cortese all'Olimpo delle più alte significazioni poetiche.

2. Prima di affrontare lo studio della riscrittura dantesca della leggenda di Traiano e del mito di Rifeo, e di entrare quindi nel campo dei rapporti intertestuali che il canto 20 stabilisce, rispettivamente, con la tradizione narrativa mediolatina e romanza, e con quella poetica classica, è bene svolgere alcune considerazioni (letterarie non teologiche ovviamente) sul problema della salvezza dei pagani virtuosi, e sulla questione connessa dell'intervento diretto di Dio nelle cose dell'uomo (che è poi la definizione tecnica di 'miracolo'). ${ }^{9}$

Dal canto 19, vv. 106-14, il lettore ha già appreso che la salvezza dei non-cristiani costituisce la rivelazione della faccia positiva della giustizia divina: la sua infinita misericordia. Cosí come la condanna dei principi falsi-cristiani (vv. 115-48) rappresenta la dimostrazione dell'aspetto negativo della stessa giustizia divina: la sua ira tremenda. Il modello al quale Dante si ispira in questo brano è naturalmente 
quello evangelico di Malteo 8.11-2: "Dico autem vobis quod multi ab oriente et occidente venient, et recumbent cum Abraham et Isaac et Iacob in regno coelorum: filii autem regni eicientur in tenebras exteriores." Se Cristo aveva sostenuto che il regno dei cieli verrà chiuso al popolo eletto e aperto ai gentili, Dante amplifica il raggio semantico del messaggio divino sostenendo che nel giorno del Giudizio il Paradiso si aprirà agli Infedeli, ai "Perse" e all'"Etiòpe," alle persone cioè che non hanno avuto sulla terra occasione di ascoltare la parola rivelata. Questi Infedeli potranno anzi leggere in Paradiso nel Libro dei Reprobi la lunga lista di coloro che dovrebbero stare vicinissimi ("prope") a Cristo, perché suoi rappresentanti sulla terra, ma che ne sono invece allontanati in eterno: la lista dei cattivi principi dell'Europa attuale, degli iniqui reggitori delle sorti dell'imperium christianum.

Nell'immediato contesto evangelico $(M t .11 .12)$ troviamo anche la citazione che fornisce il tema che viene poi sviluppato nel canto 20. Chi ha voluto avvicinarsi a Dio, anche se vissuto all'interno della cultura pagana, ha ricevuto i mezzi per poterlo fare: gli è bastato, afferma Dante, averlo desiderato ardentemente e sperato attivamente. Benché una simile conversio sembri assurda, in quanto forza le leggi stesse della giustizia divina (che richiede la fede esplicita in Cristo morto e risorto per ottenere il passaggio al Paradiso), pure durante la sua visita del cielo di Giove l'actor ha occasione di contemplare due exempla di tale miracolosa possibilità: Traiano e Rifeo. "Regnum coelorum vim patitur : et violenti rapiunt illud": per Dante la controversa frase evangelica ha un unico significato, e annuncia una verità capitale, come chiaramente indicato dalla sua citazione-parafrasi:

Regnum celorum violenza pate da caldo amore e da viva speranza, che vince la divina volontate.

Anche in absentia della fede, la pratica eccezionale del "caldo amore" (quell'amore capace di portare verso l'alto, verso la Fonte della luce), e della "viva speranza" (quella speranza che permette di intravedere una vita più autentica) possono creare le basi per l'intervento miracoloso di Dio elargente la Rivelazione, e condurre quindi alla salvazione eterna.

Come viene coinvolto l'actor/auctor nello svolgimento della grave 
tematica della salvazione dei pagani? Se la "speranza" dell'actor fin dall'inizio del suo cammino soteriologico (Inf. 1.54: "ch'io perdei la speranza de l'altezza"), è stata quella di poter concludere positivamente e felicemente l'attraversamento dei regni dell'Oltretomba, e di arrivare cosí alla finale contemplazione, facie ad faciem, dell'immagine divina; la "viva speranza" dell'auctor è quella che l'umanità possa raggiungere il suo vero obiettivo: la felicità eterna; e, di consequenza, che le venga concesso di accedere alla porta che sola può immettere a tale acquisizione: la fede nella morte e nella resurrezione di Cristo. La "viva speranza" di Dante, insomma, è che il "paganesmo" si trasformi in "cristianesmo"; realizzando cosí la vera, autentica 'metamorfosi,' che è quella cristiana del passaggio da uno stato di peccato a uno stato di grazia. La "viva speranza" di Dante è dunque non che il mondo pagano in quanto tale (rimanendo cioè pagano) venga alla fine salvato; ma che il mondo pagano riesca a trovare nella sua propria cultura quella base ideologica e conoscitiva sulla quale si possa posare la Rivelazione. Ora, una simile 'metamorfosi' si realizza al livello della narratio nel personaggio di Rifeo, qui celebrato; e al livello dell'imitatio e dell'auctoritas in quello del poeta Stazio.

Ma l'impegno diretto dell'actor/auctor nella tematica trattata si giuoca anche su un altro versante testuale. In ambedue i casi di pagani salvati in questo canto, sia quello di Traiano sia quello di Rifeo, è questione di miraculum, di intervento divino che elargisce la Rivelazione, la 'fede esplicita.' Per Dante la possibilità di salvezza in una situazione di obiettiva infidelitas non può infatti che essere frutto di una decisione divina, che opera immediatamente (come nel caso di Rifeo) o mediatamente (come nel caso di Traiano/S. Gregorio) infondendo nell'anima prescelta il dono della Grazia. Il merito da solo, le opere buone, non bastano alla salvazione: è necessario il merito divino, l'Incarnazione. La precisazione è importante, perché proprio tale punto è quello che spiega la particolare funzione rivestita da questi personaggi nel poema sacro. Nell'analisi che segue cercheremo precisamente di dimostrare come il racconto inserito della miracolosa elargizione, a Traiano o Rifeo, della grazia divina sia uno specchio nel quale il racconto-cornice del poeta-pellegrino si riflette e si conosce. Lo straordinario privilegio concesso da Dio all'anima eletta di superare una condizione di peccato originario, ha una mo- 
tivazione simile a quella vantata dall'actor: mostrare al mondo "che mal vive" le verità eterne che a Lui si riferiscono.

3. La realizzazione testuale della salvazione di Traiano nel Paradiso dantesco mantiene qualcosa della spettacolarità che essa aveva nella letteratura agiografica. ${ }^{10}$ La concessione divina all'imperatore romano della "ferma fede ... de' passi piedi" (104-5) si svolge infatti all'interno di una scenografia impregnata del gusto del 'meraviglioso cristiano' caratteristico dei miracles di Gautier de Coinci o dei milagros di Gonzalo de Berceo. Si tratta in realtà di un evento esterno e pubblico, reso universalmente noto da una folta documentazione leggendaria:

Ché l'una de lo 'nferno, u' non si riede già mai a buon voler, tornò a l'ossa; e ciò di viva spene fu mercede:

di viva spene, che mise la possa ne' prieghi fatti a Dio per suscitarla, sí che potesse sua voglia esser mossa.

L'anima gloriosa onde si parla, tornata ne la carne, in che fu poco, credette in lui che potea aiutarla; e credendo s'accese in tanto foco di vero amor, ch'a la morte seconda fu degna di venire a questo gioco.

Allo scopo di meglio comprendere questo episodio della Commedia (e l'altro ad esso collegato del 10 canto del Purgatorio) è necessario ripercorrere, anche solo nei suoi tratti essenziali, la storia della leggenda di Traiano nel Medioevo.

La leggenda della salvazione di Traiano si articola nelle fonti agiografiche medievali, cosí come nel poema dantesco, in due racconti distinti. Il primo è il resoconto di un factum memorabile: si tratta dell'aneddoto della consolazione della vedova, nel quale l'imperatore romano rinvia pressanti impegni bellici per rendere giustizia ad un'umile donna che insistentemente gliela richiede; in Traiano la preoccupazione della salus personale (cfr. Purg. 10.89-90: "I'altrui bene / a te che fia, se 'l tuo metti in oblio?"), l'esercizio altissimo della giustizia, "dovere" (92) di ogni regnante, finisce per prevalere su ogni altra cura terrena (è questo exemplum che viene riportato, 
per esaltare la virtù dell'umiltà, in Purg. 10.73-93, e che viene qui ripreso in sermo brevis per affabulare il "merto" del personaggio: "colui che . . . la vedovella consolò del figlio," 44-5). Il secondo racconto è la registrazione invece di un gesto 'meraviglioso,' del miraculum operato da un santo: papa Gregorio, colpito dall'atto di umiltà e giustizia compiuto da Traiano nei confronti dclla vedova, ottiene da Dio che l'imperatore, che pure si era reso colpevole di persecuzioni ai cristiani, "torni all'ossa," venga cioè risuscitato, per potersi pentire dei suoi peccati e convertire al cristianesimo (è questa appunto la materia narrativa che viene riproposta nell'episodio paradisiaco di cui ci stiamo occupando).

Del doppio racconto, esemplaristico e miracolistico, nel quale prende forma la leggenda di Traiano, circolano nel Medioevo varie redazioni, tutte comunque riconducibili a tre tipi fondamentali. $\mathrm{Ci}$ sono anzitutto le versioni 'agiografiche,' elaborate fra l'VIII e l'XI secolo, e inserite all'interno della Vita di S. Gregorio Magno. ${ }^{11}$ In queste Vitae (la più importante delle quali è quella del diacono Giovanni) l'attenzione è naturalmente calamitata dal potere spirituale del santo, di cui il racconto della giustizia di Traiano e della sua liberazione dalle pene dell'Inferno costituisce una delle prove più eclatanti. L'ipotesi dell'assunzione di Traiano in Paradiso, a conseguenza della negoziazione del papa con Dio, viene in questi testi categoricamente esclusa; come affermato dal diacono Giovanni: “. . . non legitur Gregorii precibus Traiani anima ab inferno liberata et in paradiso reposita, quod omnino incredibile videtur propter illud quod scriptum est: Nisi quis renatus fuerit ex aqua et Spiritu sancto non intrabit in regnum caelorum; sed simpliciter dicitur, ab inferno solummodo cruciatibus liberata." 12 Certo però che la citazione del Vangelo di Giovanni 3.3: "Nisi quis renatus fuerit. . .," offriva ai lettori della leggenda un'indicazione preziosa dello scenario dentro il quale tale salvazione poteva realizzarsi. In definitiva, ciò che nelle versioni agiografiche spiega l'interesse e l'amore di S. Gregorio per Traiano è sí l'atto di giustizia nei confronti della vedova, ma soprattutto il collegamento tipologico che tale gesto esemplare stabilisce con le parole di Isaia (1.17): "Iudicate pupillo et defendite viduam. . . ." Traiano diventa cioè agli occhi di S. Gregorio colui che ha adempiuto la parola dei profeti: diventa una figura Christi.

Esistono poi della leggenda di Traiano le versioni 'umanistiche,' 
situabili nel corso del XII secolo, $\mathrm{c}$ inserite in trattati filosofici (come la Theologia christiana di Abelardo) o politici (come il Policraticus di Giovanni di Salisbury). ${ }^{13}$ Lo scopo di queste versioni non è tanto la dimostrazione della 'santità' di Gregorio quanto l'esibizione della 'virtù' di Traiano, considerato come il migliore degli imperatori romani. Il soccorso prestato da Traiano alla vedova viene pertanto visto non più in una prospettiva tipologica (Traiano figura Christi), ma secondo un'ottica politico-simbolica (Traiano speculum principum). ̇̀ la drammatica situazione di vedovanza dell'imperium christianum (avvertita pressantemente da Giovanni di Salisbury) a richiedere urgentemente l'avvento di un principe virtuoso come Traiano, affinché essa possa essere migliorata. Il risalto assunto dalla virtus romana comporta la limitazione, in questi testi, della rilevanza presa dal $m i$ raculum cristiano, la cui espressione viene affidata a un frettoloso codicillo (sintomatico il periodo dedicatogli dal Policraticus [5.8]: "Fertur autem beatissimus papa tam diu pro eo fudisse lacrimas, donec ei in revelatione nuntiatum sit Traianum a penis inferni liberatum . .."; ${ }^{14}$ si noti come anche qui sia questione di liberazione dalle pene infernali, e non di salvazione).

Certo però che l'esaltazione umanistica della virtù di Traiano fece fare un notevole passo avanti nel cammino che porta alla sua salvazione. La soglia fra liberazione/salvazione venne comunque superata con le versioni 'scolastiche' della leggenda, circolanti durante tutto il XIII secolo (le più interessanti delle quali sono quelle offerte da Alessandro di Hales, Tommaso d'Aquino e Bonaventura). ${ }^{15}$ In queste versioni il nucleo narrativo esemplare-miracolistico della leggenda viene mortificato dall'esigenza dimostrativa e didascalica: viene funzionalizzato cioè a una questione teologica precisa; nella fattispecie: se la virtù pagana sia sufficiente a procurare la salvazione cristiana. In esse si procede inoltre a correggere le incongruenze logiche e gli errori dottrinali registrati nelle versioni agiografiche; in particolare che le pene dell'Inferno possano essere attenuate. La soluzione soteriologica dell'intricata questione teologica (Traiano, un pagano, è salvato da un miracoloso intervento di S. Gregorio), soluzione proposta dai filosofi scolastici, si basa su un'auctoritas ben più solida di quella fornita dai primitivi agiografi di papa Gregorio: cioè su un'omelia greca, attribuita a Giovanni Damasceno, della quale si cominciò ad avere notizia in Occidente verso la fine del XII secolo. 
La formulazione più coerente di questa soluzione noi la rinveniamo nella Summa Theologiae di S. Tommaso:

Praeterea, Damascenus, in eodem sermone, narrat quod Gregorius, pro Traiano orationem fundens, audivit vocem sibi divinitus illatam: Vocem tuam audivi, et veniam Traiano do. Cuius rei, ut Damascenus dicit in dicto sermone, testis est oriens omnis et occidens. Sed constat Traianum in inferno fuisse, quia multorum martyrum necem amaram instituit, ut ibidem Damascenus dicit. Ergo suffragia Ecclesiae valent etiam in inferno existentibus. ${ }^{16}$

Ciò che si evince dalla testimonianza del Damasceno è dunque che Traiano, condannato sicuramente all'inferno a causa della sua attiva infidelitas, per L'intercessione di S. Gregorio ricevette la remissione dei suoi peccati, e venne quindi salvato. Sulle modalità di realizzazione di tale salvazione S. Tommaso propone due ipotesi: la prima, più radicale, è che Traiano sia stato risuscitato in modo da potersi pentire e usufruire del dono della grazia; la seconda, più cauta, è che Traiano abbia ottenuto un rinvio del suo giudizio particolare fino al Giudizio finale:

... de facto Traiani hoc modo potest probabiliter aestimari: quod precibus beati Gregorii ad vitam fuerit revocatus, et ita gratiam consecutus sit, per quam remissionem peccatorum habuit, et per consequens immunitatem a poena. [. . .] Vel dicendum, secundum quosdam, quod anima Traiani non fuit simpliciter a reatu poenae aeternae absoluta, sed eius poena fuit suspensa ad tempus, scilicet usque ad diem iudicii.

La sospensione della sentenza nelle versioni scolastiche (caratteristica anche delle coeve raccolte di leggende, come la Legenda Aurea, dove le ipotesi della restituzione di Traiano alla vita, o quella del rinvio del giudizio divino, sono le prime fra le tante altre proposte) viene decisamente appellata dalle versioni della leggenda di Traiano in lingua volgare (sorte verso la fine del XIII e nel corso del XIV secolo). Potremmo chiamare queste versioni 'letterarie,' nel senso che esse, pur tenendo in debita considerazione la questione teologica, in realtà la superano, proiettandola in una dimensione poetica. I testi più rappresentativi sono i Fiori e vita di filosafi (26), il Novellino (69) e naturalmente la Commedia. Comune ad essi è la necessità di spazzare via le incertezze delle versioni scolastiche e di sposare la soluzione narrativamente più efficace: quella della miracolosa salvazione di Traiano. 
Significativamente la tradizione manoscritta dei Fiori ripresenta l'esitazione fra epilogo pietistico del racconto (proprio delle versioni agiografiche) e epilogo miracolistico (tipico invece delle versioni scolastiche): la maggioranza dei manoscritti (una ventina) offre infatti la prima soluzione, mentre la seconda viene sviluppata da un gruppetto di soli quattro manoscritti. Appare chiaro il fatto che l'evoluzione redazionale sia qui sintomo della trasformazione del gusto e segua il processo di letterarizzazione del testo. ${ }^{17}$

L'autore del Novellino, dal canto suo, riduce l'atto di giustizia di Traiano nei confronti della vedova a un beau geste (a una "bella cortesia"), privo di ogni implicazione trascendentale; e al tempo stesso attribuisce all'intervento miracoloso una valenza puramente mondana: "E santo Grigoro orò per lui, e dicesi per evidente miracolo che per li prieghi di questo santo papa l'anima di questo imperadore fu liberata dalle pene de l'inferno, e andone in vita eterna; ed era stato pagano." Ciò che gli preme enfatizzare, infatti, è da un lato il magnifico spettacolo del trionfo della giustizia e del miracolo cristiano, dall'altro la sua brillante traduzione linguistica: il racconto non a caso si sigilla con una squisita figura retorica, nell'opposizione ossimorica di vita eterna e pagano. ${ }^{18}$

Decisivo e definitivo per la storia di questo tema, cosí come di tanti altri temi dell'immaginario medievale, l'apporto dantesco. La Commedia intende scoprire la veritas del destino eterno di Traiano; ciò che coinvolge un dialogo serrato e polemico con le 'fonti': la correzione dei loro errori e la risposta alle loro domande. Credo che sia pertanto irrilevante porsi il problema della 'fonte' specifica secondo la quale Dante avrebbe costruito quest'episodio, poiché in realtà egli ha scrutato l'intero ventaglio delle 'fonti' pertinenti per offrircene un bilancio. La novitas del messaggio poetico dantesco sarà allora rinvenibile attraverso un confronto con la serie di testi già evocata.

Rispetto alle versioni precedenti della leggenda, agiografiche umanistiche e scolastiche, versioni tutte caratterizzate da un atteggiamento distaccato nei confronti del fatto narrato e da una finalità didascalica, Dante, portando avanti il processo di contemporaneizzazione dell'exemplum iniziato dalle versioni novellistiche, fa di Traiano un mito personale, gli attribuisce una funzione autoconoscitiva. Dante, in altre parole, avvicina la conversio del personaggio paradigmatico 
alla sua propria conversio, la cui descrizione noi vediamo affidata ai primi due canti dell'Inferno. ${ }^{19} \mathrm{Nel}$ contesto specifico, la figura di Traiano viene a rafforzare il significato assunto da quelle di David e Ezechia, di cui abbiamo già discusso.

E in realtà, la storia dell'imperatore che visse due volte, la prima da pagano e la seconda da cristiano, arrivando a sperimentare in tal modo sia la realtà infernale sia la realtà paradisiaca (vv. 47-8: "per l'esperienza / di questa dolce vita e de l'opposta"), si ripete esattamente nel pattern narrativo dell'actor della Commedia che, a metà della sua vita, si ritrova nella selva della lontananza da Dio per iniziare il suo cammino verso la reintegrazione eterna: cammino che lo condurrà a visitare prima il regno della dannazione $\mathrm{e}$ poi quello della salvazione. Cosí come la vicenda della miracolosa rinascita di Traiano alla vita della grazia, giustificata dal proprio "merto" (l'essere simbolo della giustizia terrena) e portata a effetto dall'intercessione di un santo, riflette specularmente la vicenda del personaggio-poeta, che per intervento miracoloso di Dio e per intercessione delle "tre donne benedette" (Inf. 2.124), si vede affidato (sulla base dell'esperienza amorosa consegnata al libello giovanile della Vita Nuova) il compito di combinare il viaggio classico di Enea col viaggio cristiano di S. Paolo nell'unico viaggio della cristianità decaduta verso la definitiva palingenesi.

4. Un'indicazione ermeneutica di grande rilievo per poterci spiegare la ragione profonda che sta dietro la scelta di Rifeo a completare il sestetto dei principi giusti ci viene da una discrepanza fra Convivio e Commedia riguardante le omologie storiche stabilite fra mondo ebraico-cristiano e mondo classico. ${ }^{20}$ Mentre infatti il Convivio propone una correlazione fra il re David, iniziatore della "progenie santissima" che conduce a Cristo, e Enea, iniziatore della progenie imperiale (4.5.6: "E tutto questo fu in uno temporale, che David nacque e nacque Roma, cioè che Enea venne di Troia in Italia, che fu origine de la cittade romana, sí come testimoniano le scritture"); la Commedia istituisce una ben diversa corrispondenza fra lo stesso re David e Rifeo: personaggio che passa cosí a simbolizzare il nuovo padre spirituale dell'imperium christianum. Interpreto tale divario come l'indicazione del passaggio da una fase 'umanistica' della cultura di Dante (rappresentata dall'enciclopedia filosofica del Convivio), da 
un momento cioè nel quale Dante crede alla continuità fra mondo classico e mondo cristiano; a una fase per cosí dire 'agostiniana' (rappresentata dall'cnciclopedia poctica della Commedia), a un momento cioè nel quale Dante si rende conto dello sbarramento esistante fra mondo classico e mondo cristiano (significato dall'Incarnazione), e vede di conseguenza il rapporto fra mondo classico e mondo cristiano in termini di discontinuità e frattura. Se nel Convivio l'Eneide viene considerata alla stregua delle "scritture" sacre, e Virgilio alla stregua dei profeti del Vecchio Testamento, nella Commedia invece l'Eneide è la premonizione di una verità che va glossata, l'annuncio di un senso cristiano che va integrato (come dimostrato dall'episodio di Stazio), cosí come Virgilio è l'auctor della tragedia per eccellenza dell'Antichità, dell'opera cioè che affabula non la conclusione positiva dell'iter conoscitivo dell'uomo (come appunto la Comedia dantesca), ma la conclusione negativa. Né Virgilio né Enea furono infatti liberati dal Limbo, al momento del descensus di Cristo nell'Inferno. Anzi, la ripetizione poetica di tale descensus, il poema sacro, accerta la liberazione dell'anti-Enea, di Rifeo, e l'incoronazione dell'anti-Virgilio, di Dante.

Anche sul conto di Rifeo la Commedia intende apporre la glossa definitiva; tanto più memorabile quanto più essa è nascosta e imprevedibile:

Chi crederebbe giú nel mondo errante che Rifëo Troiano in questo tondo fosse la quinta de le luci sante?

Ora conosce assai di quel che 'I mondo veder non può de la divina grazia, ben che sua vista non discerna il fondo.

L'interrogazione iniziale che troviamo nella sestina dedicata alla presentazione di Rifeo, semplice comparsa nell'Eneide, serve sí ad enfatizzare il senso di stupita meraviglia che suscita la lieta novella della sua salvazione nel lettore cristiano, ma vuole soprattutto accentuare la straordinaria novitas del messaggio poetico dantesco, che corregge in modo tanto rivoluzionario il testo virgiliano. Nel secondo libro dell'Eneide troviamo Rifeo fra i compagni più valorosi di Enea che tentano di opporre un'ultima disperala difesa contro la forza soverchiante dei Greci; finché lo vediamo cadere sopraffatto dai nemici: 
"cadit et Riphaeus, iustissimus unus / qui fuit in Teucris et servantissimus aequi / (dis aliter visum)" (426-8); mentre d'altro canto Enea è destinato a uscire vivo dalla battaglia: "Danaum et, si fata fuissent / ut caderem, meruisse manu" (434-5). La documentazione dantesca per quanto riguarda la fictio, il livello istoriale dell'episodio, è tutta ricavata da questi pochi versi virgiliani. Sulla base di tale auctoritas, Dante assume Rifeo a prototipo della giustizia umana; partendo cioè dall'affermata presenza in lui, al grado superlativo ("iustissimus"), della stessa virtù che caratterizza il suo compagno più fortunato, Enea; presenza che assume i toni del rito religioso ("servantissimus aequi"). Del tutto nuova è invece la glossa che Dante appone alla littera del poema classico. L'elaborazione della sententia, del senso allegorico, non trova infatti nessun avallo nella lettura secolare di Virgilio, nei commenti cioè e nelle glosse medievali. La storia della conversio di Rifeo, affabulata nel secondo discorso dell'aquila, ci viene presentata nel silenzio totale dell'esegesi cristiana:

L'altra, per grazia che da sí profonda fontana stilla, che mai creatura non pinse l'occhio infino a la prima onda,

tutto suo amor là giù pose a drittura: per che, di grazia in grazia, Dio li aperse l'occhio a la nostra redenzion futura;

ond' ei credette in quella, e non sofferse da indi il puzzo più del paganesmo; e riprendiene le genti perverse.

Quelle tre donne li fur per battesmo che tu vedesti da la destra rota, dinanzi al battezzar piú d'un millesmo.

Mentre al livello della costruzione narrativa dell'episodio Dante si attiene scrupolosamente alle informazioni che gli provengono dal testo classico, al livello della costruzione allegorica procede ad una radicale correzione dello stesso testo. Dall'imitatio passa all'aemulatio. L'intervento correttorio tocca in particolare l'inciso del testo di partenza: "Dis aliter visum." Mentre infatti Virgilio riconosce nella morte di Rifeo, e nella sua consequente esclusione dal viaggio fatale di Enea verso Roma, una decisione avversa degli dei; Dante attribuisce a quella stessa morte un significato positivo, interpretandola come il passaggio verso la vera patria, verso la cittadinanza 
paradisiaca. La comedia cristiana diventa cosí l'inveramento della tragedia classica; e al tempo stesso il viaggio dell'actor fino alla visione finale di Dio si pone in una relazione di differenziazione rispetto al viaggio di Enea verso la Roma terrena (la città "dove Cristo tutto di si merca"), e in rapporto di identificazione rispetto alla peregrinatio di Rifeo verso "quella Roma onde Cristo è romano." La creazione del nuovo mito di Rifeo assumerà allora il valore non di un postremo omaggio alla poesia di Virgilio (come suona il commento tradizionale), ma della resa finale dei conti poetici fra Eneide e Commedia.

McGill University

\section{NOTE}

* Testo di una lectura tenuta a Napoli il 3 febbraio 1988 nel contesto della Lectura Dantis Neapolitana diretta da Pompeo Giannantonio.

1 Sul significato della figurazione dell'aquila nella Commedia (simbolo non solo dell'impero, ma anche dell'"altezza d'ingegno" del poeta-pellegrino) si veda ora Brugnoli 169-86.

2 Delle non numerose interpretazioni di questo canto si sono soprattutto tenute presenti nella nostra lectura quelle di Paratore e di Pézard. Spunti interessanti si trovano nei cappelli introduttivi dei commenti di Bosco-Reggio e di Pasquini-Quaglio.

3 Su questo punto si veda Paratore 293-4.

4 Per un'analisi complessiva del canone dei principi giusti si può ricorrere al lavoro di Renaudet 202-20.

5 Si noti l'eco intratestuale dell'analoga operazione compiuta dal Veltro, ma in senso negativo: "questi la caccerà per ogne villa" (Inf. 1.109).

6 Sul valore di questa distinzione si può ora consultare il lavoro di Minnis, soprattutto $103-12$.

7 Si veda 1'"Introduzione alla Monarchia di Dante," ora inclusa come prefazione al testo contenuto nel secondo tomo delle Opere minori di Dante, 241-69.

8 Testi fiorentini del Dugento 93.

9 Per questa complessa e dibattuta questione si rinvia il lettore alla convincente trattazione fornita da Foster 156-253.

10 Sulla diffusione della figura di Traiano nella cultura medievale verte l'ottimo lavoro di Whatley. Si vedano ora anche le pagine di Vickers.

11 Whatley 27-31.

12 Parrologia lasina 75: 105-6.

13 Whatley 31-6.

14 Policraticus 5.8: p. 315 nell'edizione a cura di Webb. 
15 Whatley $36-43$.

16 Si tratta della Quaestio 71 ("Utrum suffragia prosint existentibus in inferno"), a.15, del Supplementum alla Tertia Pars.

17 Fiori e vita di filosafi 33-9.

18 Per l'interpretazione di questa novella si vedano Mulas e Paolella 121-5.

19 Per questa prospettiva ermeneutica si rinvia a Freccero.

20 Su Rifeo, oltre alla bibliografia citata nella nota 2 , si può vedere la voce dell'Enciclopedia dantesca, firmata da Giorgio Padoan.

\section{OPERE CITATE}

Alighieri, Dante. La Commedia secondo l'antica vulgata. 4 voll. A cura di Giorgio Petrocchi. Milano: Mondadori, 1966-67.

- Opere minori. Tomo 2. A cura di Vincenzo Mengaldo et al. Milano: Ricciardi, 1979.

Bosco, Umberto e Giovanni Reggio (a cura di). La Divina Commedia. 3 voll.

Firenze: Le Monnier, 1979.

Brugnoli, Giorgio. Per suo richiamo. Pisa: Opera Universitaria, 1981.

D'Agostino, Alfonso (a cura di). Fiori e vita di filosafi e d'altri savi e d'imperadori. Firenze: La Nuova Italia, 1979.

Foster, Kenelm. The Two Dantes and Other Studies. Berkeley and Los Angeles: University of California Press, 1977.

Freccero, John. Dante. The Poetics of Conversion. Cambridge, Mass.: Harvard UP, 1986.

Giovanni di Salisbury. Policraticus. Ed. Clement C. J. Webb. Vol. 1. Oxford: Clarendon Press, 1909.

Minnis, A. J. Medieval Theory of Authorship. London: Scholar Press, 1984.

Mulas, Luisa. Lettura del Novellino. Roma: Bulzoni, 1984.

Padoan, Giorgio. "Rifeo." Enciclopedia dantesca 4: 923-24.

Paolella, Alfonso. Retorica e racconto. Argomentazione e finzione nel Novellino.

Napoli: Liguori, 1987.

Paratore, Ettore. Tradizione e struttura in Dante. Firenze: Sansoni, 1968.

Pasquini, Emilio e Antonio Quaglio (a cura di). La Commedia. 3 voll. Milano: Garzanti, 1986.

Pézard, André. "Riphée ou la naissance d'un mythe." Revue des Études italiennes 25 (1979): 5-40.

Renaudet, Augustin. Dante humaniste. Paris: Les Belles Lettres, 1952.

Schiaffini, Alfredo (a cura di). Testi fiorentini del Dugento. Firenze: Sansoni, 1954.

Vickers, Nancy. "Seeing is Believing: Gregory, Trajan, and Dante's Art." Dante Studies 101 (1983): 67-85.

Whatley, Gordon. "The Uses of Hagiography: The Legend of Pope Gregory and the Emperor Trajan in the Middle Ages." Viator 15 (1984): 25-63. 